\title{
Deep-vein thrombosis detection rates and consideration of the living environment in a tsunami disaster area during the disaster reconstruction phase: A cross-sectional study
}

Hidenori Onishi', Osamu Yamamura ${ }^{2}$, Shinsaku Ueda ${ }^{3}$, Muneichi Shibata ${ }^{4}$, Soichi Enomoto ${ }^{5}$, Fumie Maeda ${ }^{6}$, Hiromasa Tsubouchi ${ }^{7}$,Takeshi Hirobe ${ }^{8}, S^{2}$ adao Shimizu', Takahiro Kishimoto ${ }^{10}$, Hiroshi Chiba', Kazuhiro Sasaki ${ }^{12}$, Kazuhiko Hanzawa' ${ }^{13}$ Tadanori Hamano ${ }^{14}$, Yasunari Nakamoto ${ }^{15}$, Bunji Kaku' ${ }^{16}$, Hidekazu Terasawa ${ }^{17}$

'Department of Community Medicine, Faculty of Medical Science, University of Fukui, Fukui, Japan

${ }^{2}$ Department of Community Medicine, Faculty of Medical Science, University of Fukui and Second Department of Internal Medicine, Faculty of Medical Science, University of Fukui, Fukui, Japan

${ }^{3}$ Department of Thoracic Surgery, Ishinomaki Red Cross Hospital, Miyagi, Japan

${ }^{4}$ Department of Cardiology, Mackay Base Hospital, Queensland, Australia

${ }^{5}$ Second Department of Internal Medicine, Faculty of Medical Science, University of Fukui, Japan

${ }^{6}$ Department of Clinical Laboratory, University of Fukui, Fukui, Japan

${ }^{7}$ Department of Radiotechnology, Fukui Ken Saiseikai Hospital, Fukui, Japan

${ }^{8}$ Department of Clinical Laboratory, Fukui Prefectural Hospital, Fukui, Japan

'Department of Medical Laboratory, National Hospital Organization Kanazawa Medical Center, Ishikawa, Japan

${ }^{10}$ Department of Radiology, University of Fukui Hospital, Fukui, Japan

"Division of Clinical Laboratory, Morioka Municipal Hospital, Iwate, Japan

${ }^{12}$ Department of Neurology, Morioka Municipal Hospital, Iwate, Japan

${ }^{13}$ Department of Respiratory Surgery, Graduate School of Medicine, University of Niigata, Niigata, Japan

${ }^{14}$ Second Department of Internal Medicine, Faculty of Medical Science, University of Fukui, Fukui, Japan

${ }^{15}$ Second Department of Internal Medicine, Faculty of Medical Science, University of Fukui, Fukui, Japan

${ }^{16}$ Department of Cardiology, Toyama Red Cross Hospital, Toyama, Japan

${ }^{17}$ Department of Community Medicine, Faculty of Medical Science, University of Fukui, Fukui, Japan

\section{Abstract}

Introduction: Tsunami victims of the Great East Japan Earthquake were screened for deep-vein thrombosis (DVT) in order to compare the DVT incidence rates between temporary and non-temporary housing resident groups.

Material and methods: Lower extremity venous ultrasonography was performed on 290 subjects (64 men and 226 women; mean age $=71.9 \pm 7.9$ years) at 44 months after the disaster. All subjects completed questionnaires to gather information about their background factors which included the Kessler Psychological Distress Scale: K6.

Results: The DVT detection rate was 10.7\% in the temporary group. In the non-temporary group, it was I I.3\% among the subjects who previously lived in temporary housing. For the subjects who were living in their own homes,

Address for correspondence: Osamu Yamamura, Department of Community Medicine, Faculty of Medical Science, University of Fukui and Second Department of Internal Medicine, Faculty of Medical Science, University of Fukui, 23-3 Matsuokashimoaizuki Yoshida-gun Eiheiji-cho Fukui 9I0-II04 Japan, +8I 776-6I-8264, e-mail: kapi@u-fukui.ac.jp 
it was 9.2\%. Psychological distress levels measured by $\mathrm{K} 6$ were significantly higher in the temporary housing group than in the non-temporary housing group. The multivariate analysis showed that the background factor associated with DVT risk was SV (soleal vein) dilatation in all subjects as well as in the non-temporary housing group, while hypertension and use of sleeping pills were found to be the factors in the temporary housing group. Conclusions: DVT detection rates were similar between the temporary and non-temporary housing groups, and were higher than that in the Japanese general population. The psychological distress level of the tsunami victims measured by $\mathrm{K} 6$ was also higher in the temporary housing group than in the non-temporary housing group. It is necessary to establish a long-term and awareness-raising disaster victim support system.

Key words: Great East Japan Earthquake, tsunami disaster area, temporary housing, disaster-related diseases, psychological distress survey

Acta Angiol 2020; 26, 4: 129-139

\section{Introduction}

After a major disaster, the risk of cardiovascular disease, infection and psychological disorders increases among survivors under stressful conditions [I]. Deep vein thrombosis (DVT), one of the disaster-related diseases, has been attracting public attention since the Mid Niigata Prefecture Earthquake in 2004 [2]. An increased risk of developing DVT was reported among evacuees in emergency shelters and temporary housing in disaster areas [2-6]. Post-disaster DVT is assumed to occur at a high rate due to poor living conditions in shelters and temporary housing, under which evacuees dehydrate because they try to refrain from water intake to reduce their frequency of urination, face low levels of physical activity and sleep in vehicles [2-6].

In Ishinomaki City, Miyagi Prefecture, another area affected by a tsunami after the Great East Japan Earthquake, an increased risk of developing DVT over a long period of time was reported among residents in temporary housing and houses in the affected area even after their living environment was improved [4]. Our study was conducted in Watari-gun, Miyagi Prefecture (Watari-town and Yamamoto-town), an area which was also severely affected by the tsunami. In our study area, the temporary housing was gradually removed as reconstruction progressed from the third year after the disaster, and the living conditions for survivors were improved. DVT screening was performed for the purpose of prevention and to raise awareness among disaster survivors. Temporary housing residents are more likely to develop DVT because of their low activity levels. This study reports on the comparison of DVT detection rates between temporary housing residents (temporary housing group) and non-temporary housing residents (non-temporary housing group) and also on the identified risk factors.

\section{Material and methods}

\section{Disaster situation in our study area}

Watari-gun, Miyagi Prefecture (Watari-town and Yamamoto-town) is located approximately $40 \mathrm{~km}$ south of Sendai City and is an industrial area focusing on agriculture and fishing with a population of approximately 50,000. In the Great East Japan Earthquake, there were 885 people who died or were missing, 13,000 people were forced to evacuate from their homes, and 7,075 houses were destroyed or partially destroyed. In September 201I, the number of evacuees living in temporary housing reached 6,050 . These temporary housing complexes were however mostly removed as reconstruction progressed.

\section{Study subjects}

A total of 290 subjects who were living in temporary housing and houses in neighboring areas in Watari-gun, Miyagi Prefecture (64 men, 226 women; mean age = $=71.9 \pm 7.9$ years) participated in our screening activity. The subjects were divided into two groups: 149 in the temporary group (33 men, 116 women; mean age $=73.4 \pm 8.4$ years) and $14 \mathrm{I}$ in the non-temporary group ( 31 men, 110 women; mean age $=70.3 \pm$ \pm 7.0 years). Of the $|4| \mathrm{I}$ subjects in the non-temporary housing group, 44 were those who previously lived in temporary housing $(9$ men, 35 women; mean age $=$ $=70.8 \pm 9.4$ years), and 97 were those who continued living in their own homes (22 men, 75 women; mean age $=70.0 \pm 5.6$ years). There were 179 subjects who previously underwent DVT screenings (including screenings conducted by other organizations). The screening was performed at 44 months after the disaster for a total of 2 days (November I and 2, 20I4). Two subjects in the temporary housing group and four in the non-temporary housing group were excluded because 
of inadequacies in the Kessler Psychological Distress Scale (K6) questionnaire, resulting in the temporary housing group having 147 and the non-temporary housing group having 137. Our targeted number of participants was approximately 300.

\section{Setting and examination team}

DVT screening was undertaken at meeting places located in temporary housing, healthcare centers and public halls in Yamamoto-town, Watari-gun, Miyagi Prefecture. The medical examination team was organized mainly by the University of Fukui School of Medical Sciences with cooperation from volunteer healthcare providers (doctors, nurses, laboratory medical technologists, etc.) and medical students.

\section{Examination items}

Our DVT screening activity was performed in temporary housing in cooperation with the local authority of Watari-town and Yamamoto-town. Posters to raise awareness about DVT prevention were displayed one month prior to the screening. Participation in the screening was on a voluntary basis. All subjects provided written informed consent, and the examination was conducted in the following order: a questionnaire (age, sex, lifestyle, K6, underlying disease, life after a disaster), blood pressure measurement, lower-extremity venous ultrasonography and explanation of the result. The underlying diseases (dyslipidemia, diabetes mellitus, heart disease, hypertension) of the subjects were reported based on their answers to the self-report questionnaire. When subjects answered in the questionnaire that the time spent walking was reduced in comparison with before the disaster, they were classified as having reduced walking hours. The ultrasonography was performed by several medical technologists, each with more than five years of experience $[5,6]$. To improve uniformity, the evaluation criteria were discussed prior to the screening $[5,6]$. Screening was undertaken using portable ultrasound machines (LOGIQe, GE Healthcare with a 3.3-10.0 $\mathrm{MHz}$ linear probe; Noblus, Hitachi Medical Corporation with a 5-18 MHz linear probe; CX50, Philips Japan, Ltd., with a 3-12 MHz linear probe; Viamo, Canon Medical Systems Corporation with a $6.2-11.0 \mathrm{MHz}$ linear probe; NanoMaxx, SonoSite Inc., with a 6-13 $\mathrm{MHz}$ linear probe). Ultrasound examinations were performed from the calf to the popliteal veins in the sitting position $[5,6]$. Only screening of the calf to the popliteal veins was conducted due to the large number of participants as well as the limitations in our screening setting and time $[5,6]$. The presence of thrombus was confirmed using a combination of the color Doppler and the compression technique, where there was a lack of compressibility [5-8]. Thrombi were classified as or- ganized or fresh [6, 9-II]. Inner diameters (short-axis) of the soleal vein (SV) were measured in the sitting position with the lower extremity muscles relaxed, and maximum diameter was used in our analysis. When measuring the diameters, the probe was placed so as not to put pressure on the SV and measurements were not made on the venous valves. The maximum diameter of the soleal vein (SV) was examined, and a diameter $\geq 8 \mathrm{~mm}$ was defined as dilatation $[6,12]$. Subjects who were found to be DVT-positive received a patient referral document and were encouraged to visit their local medical institutions. $\mathrm{K} 6$ was also used to measure the psychological distress levels of the subjects. K6 consisted of six items measuring depression and anxiety over the past month on a five-point scale [13]. The Japanese version of the $\mathrm{K} 6$ has also been developed and the reliability and validity have been confirmed [14]. Because the levels of psychological distress caused by the tsunami disaster were assumed to be high in our study area, subjects who scored more than 13 points were classified as having a psychological disorder [15].

\section{Statistical analysis}

Age, systolic blood pressure, diastolic blood pressure, $\mathrm{K} 6$ and SV diameter are reported as means \pm standard deviations, and categorical variables are reported as frequency and percentage (\%). R commander version I.28 was used for the statistical analysis. Mann-Whitney $U$ test and the $\chi^{2}$ test (including Yates' continuity correction) were used for comparisons between the two groups. To identify risk factors for DVT, multivariate logistic regression analysis (stepwise method) was performed. In all comparative tests, a p-value of $<0.05$ was considered statistically significant.

\section{Results}

DVT was diagnosed in 30 of the 290 subjects screened (10.3\%), of whom 13 were fresh thrombi (4.4\%) and 17 were organized thrombi $(5.8 \%)$.

Comparison of demographic characteristics in all subjects with or without DVT is shown in Tables I, 2 and Figure I.

Univariate analysis showed that SV dilatation (43.3\% vs 19.2\%; $p<0.01$ ) and a previous history of DVT screening (83.3\% vs $59.2 \%$; $p<0.05)$ were significantly higher in the DVT-positive group than in the negative group. Multivariate analysis showed that SV dilatation was the background factor associated with DVT risk in all subjects (odds ratio 3.21 ; $95 \%$ confidence interval I.460-7.040; $p=0.0035$ ).

Comparison of demographic characteristics of subjects with or without DVT in the temporary housing group is shown in Tables I, 2, and Figure I. 


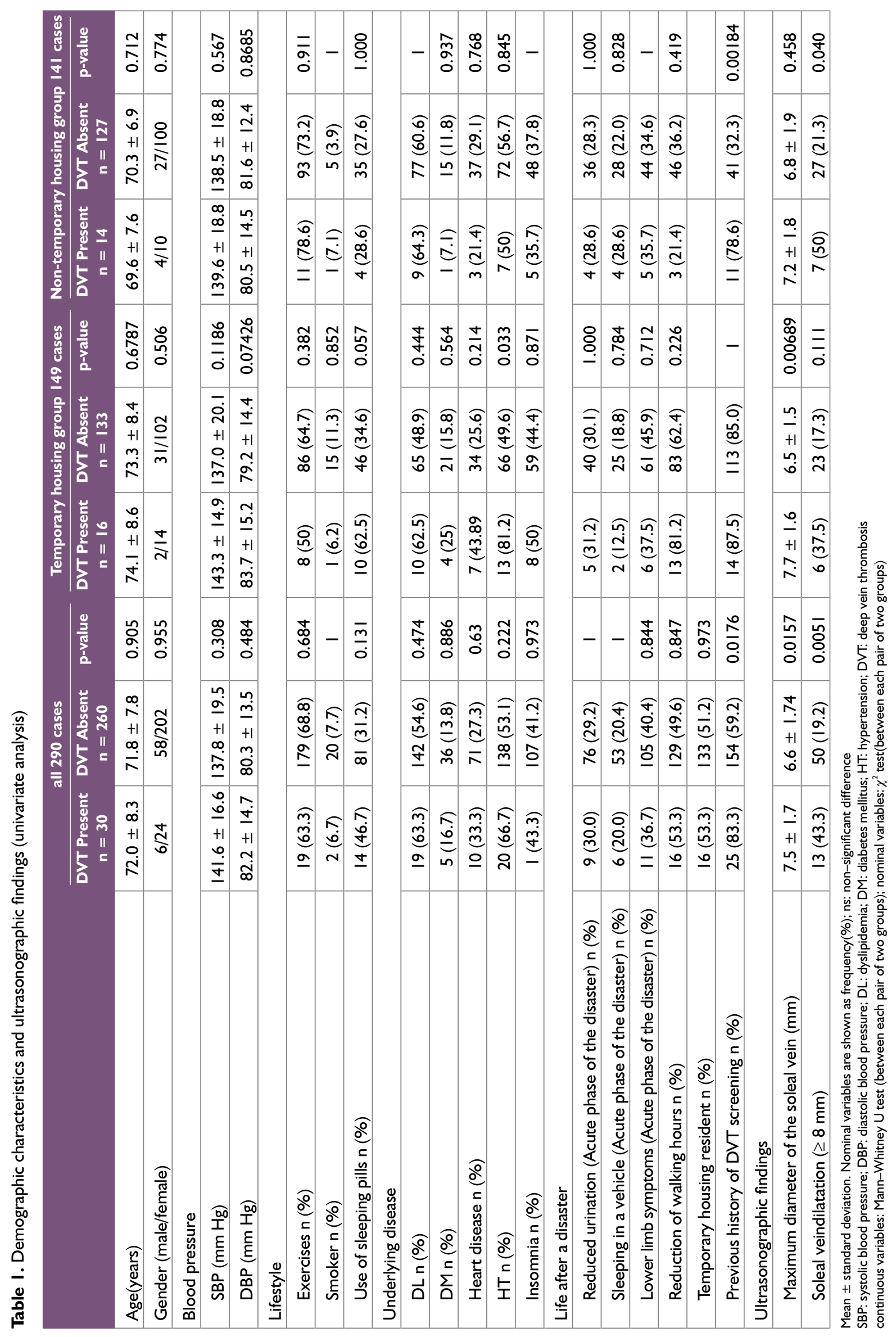




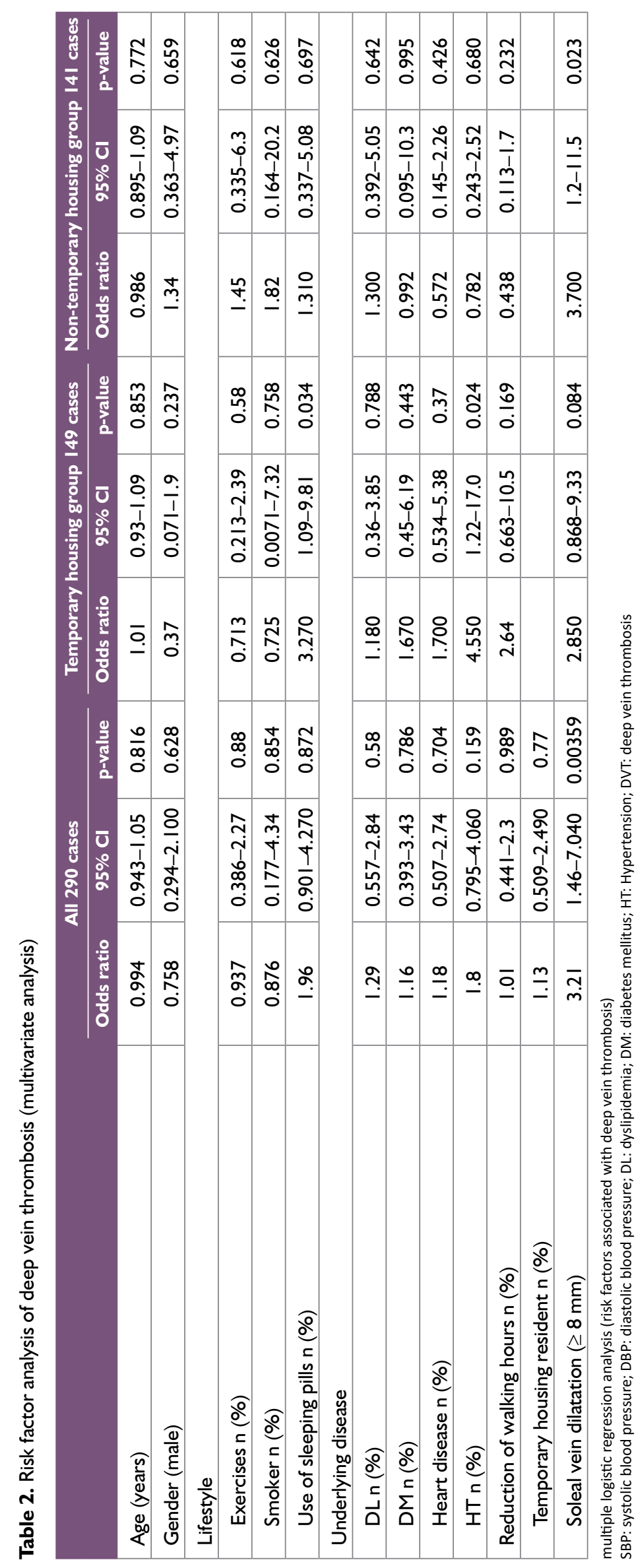




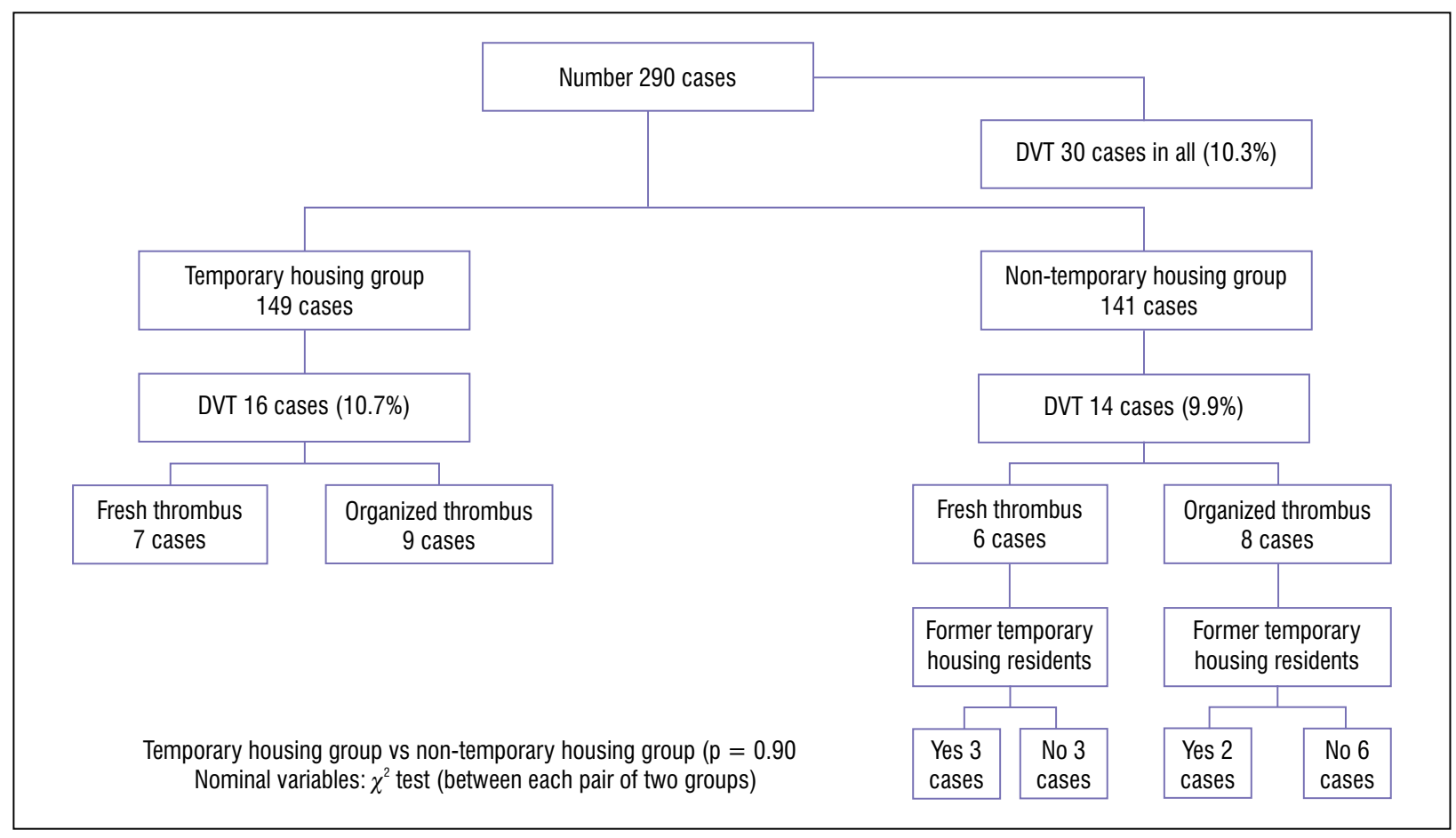

Figure I. Flow diagram of subject selection

Univariate analysis showed that the rate of subjects with hypertension $(81.2 \%$ vs $49.6 \%$; $p<0.05)$ was significantly higher in the DVT-positive group than in the negative group. Multivariate analysis showed that hypertension (odds ratio 4.4; $95 \%$ confidence interval I.200-16.100; $p=0.025$ ) and use of sleeping pills (odds ratio 3.27; 95\% confidence interval I.090-9.8I0; $p=0.034$ ) were the risk factors for DVT in the temporary housing group.

Comparison of demographic characteristics of subjects with or without DVT in the non-temporary housing group is shown in Tables I, 2, and Figure I.

Univariate analysis showed that SV dilatation $(50.0 \%$ vs $21.3 \% ; p<0.05)$ and previous history of DVT screening $(78.6 \%$ vs $32.3 \% ; p<0.01)$ were significantly higher in the DVT-positive group than in the negative group. Multivariate analysis showed that SV dilatation (odds ratio 3.7; 95\% confidence interval I.200-II.500; $\mathrm{p}=0.023$ ) was the background factor associated with DVT risk in the non-temporary housing group.

Comparison of DVT detection rates and background factors between temporary and non-temporary housing groups is shown in Table 3 and Figure 1 .

The DVT detection rates in the temporary and non-temporary housing groups were $10.7 \%$ ( 16 of 149) and $9.9 \%$ (I4 of I4I), respectively, Therefore, no significant difference was observed $(p=0.90)$. Of the I6 DVT-positive subjects in the temporary housing group, seven had fresh thrombi and nine had organized thrombi. The DVT detection rate for the former temporary housing residents in the non-temporary housing group was $11.3 \%$ (5 of 44). Of the five, three had fresh thrombi and two had organized thrombi. The DVT detection rate among those who continued living in their homes in the non-temporary group was $9.2 \%$ (9 of 97). Of the nine, three had fresh thrombi and six had organized thrombi. In the comparison of background factors, age $(73.4 \pm 8.4$ years vs $70.3 \pm 7.0$ years; $p<0.005)$, reduction of walking hours $(64.4 \%$ vs $34.8 \% ; p<0.0001$ ) and previous history of DVT screening $(85.2 \%$ vs $36.9 \%$; $p<0.000$ I) were significantly higher in the temporary housing group than in the non-temporary housing group.

Comparison of psychological distress levels of subjects between temporary and non-temporary housing groups is shown in Table 4.

Univariate analysis showed that age $(73.3 \pm 8.4$ years vs $70.3 \pm 7.0$ years; $\mathrm{p}<0.005), \mathrm{K} 6$ ( II $.2 \pm 5.9$ vs $9.7 \pm 4.9 ; p<0.05)$ and psychological disorder (38.1\% vs $22.6 \%$; $p<0.01$ ) were significantly higher in the temporary housing group than in the non-temporary housing group. 
Table 3. Comparison of temporary housing and non-temporary housing group (characteristics of individuals studies)

\begin{tabular}{|c|c|c|c|}
\hline & $\begin{array}{l}\text { Temporary housing } \\
n=149\end{array}$ & $\begin{array}{l}\text { Non-temporary housing } \\
\qquad n=141\end{array}$ & p-value \\
\hline Age (years) & $73.4 \pm 8.4$ & $70.3 \pm 7.0$ & 0.00276 \\
\hline Gender (male/female) & $33 / 116$ & $31 / 110$ & 1 \\
\hline \multicolumn{4}{|l|}{ Blood pressure } \\
\hline $\mathrm{SBP}(\mathrm{mm} \mathrm{Hg})$ & $137.7 \pm 19.7$ & $138.7 \pm 18.7$ & 0.6483 \\
\hline $\mathrm{DBP}(\mathrm{mm} \mathrm{Hg})$ & $79.7 \pm 14.5$ & $81.4 \pm 12.6$ & 0.1243 \\
\hline \multicolumn{4}{|l|}{ Lifestyle } \\
\hline Exercises n (\%) & $94(63.1)$ & $104(73.8)$ & 0.0679 \\
\hline Smoker n (\%) & $16(10.7)$ & $6(4.3)$ & 0.0626 \\
\hline Use of sleeping pills n (\%) & $56(37.6)$ & $39(27.7)$ & 0.094 \\
\hline \multicolumn{4}{|l|}{ Underlying disease } \\
\hline DL n (\%) & $75(50.3)$ & $86(6 I)$ & 0.0878 \\
\hline DM n (\%) & $25(16.8)$ & $16(11.3)$ & 0.247 \\
\hline Heart disease n (\%) & $4 \mathrm{I}(27.5)$ & $40(28.4)$ & 0.976 \\
\hline HT n (\%) & $79(53.0)$ & $79(56)$ & 0.692 \\
\hline Insomnia n (\%) & $67(45)$ & $53(37.6)$ & 0.871 \\
\hline \multicolumn{4}{|l|}{ Life after a disaster } \\
\hline Reduced urination (Acute phase of the disaster) $n(\%)$ & $45(30.2)$ & $40(28.4)$ & 0.831 \\
\hline Sleeping in a vehicle (Acute phase of the disaster) $n(\%)$ & $27(18.1)$ & $32(22.7)$ & 0.412 \\
\hline Lower limb symptoms (Acute phase of the disaster) n (\%) & $67(45)$ & $49(34.8)$ & 0.098 \\
\hline Reduction of walking hours $\mathrm{n}(\%)$ & $96(64.4)$ & $49(34.8)$ & $<0.0001$ \\
\hline Previous history of DVT screening n (\%) & $127(85.2)$ & $52(36.9)$ & $<0.0001$ \\
\hline \multicolumn{4}{|l|}{ Ultrasonographic findings } \\
\hline DVT n (\%) & $16(10.7)$ & $14(9.9)$ & 0.973 \\
\hline Maximum diameter of the soleal vein $(\mathrm{mm})$ & $6.6 \pm 1.5$ & $6.9 \pm 1.9$ & 0.185 \\
\hline Soleal vein dilatation $(\geq 8 \mathrm{~mm})$ & $29(19.5)$ & $34(24.1)$ & 0.414 \\
\hline
\end{tabular}

Mean \pm standard deviation. Nominal variables are shown as frequency $(\%)$.

SBP: systolic blood pressure; DBP: diastolic blood pressure; DL: dyslipidemia; DM: diabetes mellitus; HT: hypertension; DVT: deep vein thrombosis

Continuous variables: Mann-Whitney $U$ test (between each pair of two groups); nominal variables: $\chi^{2}$ test (between each pair of two groups)

Table 4. Comparison of temporary housing and non-temporary housing group (Kessler Psychological Distress Scale K6)

\begin{tabular}{|l|c|c|c|}
\hline & Temporary housing group $n=147$ & Non-temporary housing group $n=137$ & $p$-value \\
\hline Age (years) & $73.3 \pm 8.4$ & $70.1 \pm 6.9$ & 0.0028 \\
\hline Gender (male/female) & $33 / 114$ & $31 / 106$ & 1 \\
\hline K6 & $11.2 \pm 5.9$ & $9.7 \pm 4.9$ & 0.0264 \\
\hline K6 $(\geq 13)$ & $56(38.1)$ & $31(22.6)$ & 0.007 \\
\hline
\end{tabular}

Mean \pm standard deviation. Nominal variables are shown as frequency $(\%)$

Continuous variables: Mann-Whitney $U$ test (between each pair of two groups) nominal variables $\chi^{2}$ test (between each pair of two groups)

\section{Discussion}

\section{DVT detection rates and risk factors} in all subjects

A high incidence of DVT detected by the lower extremity venous ultrasonography was also reported in a study conducted among residents in the affected areas after the Mid Niigata Prefecture Earthquake in 2004, and the incidence rate remained higher compared with that in the non-disaster area in Japan even eight years after the disaster [16]. The high detection rate (13.5\%) was also reported in the temporary housing resident population 
in the case of Iwate Prefecture even four years after the Great East Japan Earthquake. Therefore, the risk of DVT is assumed to increase after a major disaster regardless of the type of evacuation, and is likely to remain high for a long period of time [5]. In this study, the overall DVT detection rate at 44 months after the disaster was $10.3 \%$, which was still much higher than that of the general Japanese population (1.8-2.3\%) [4]. Our screening program has also identified that SV dilatation is a background factor associated with DVT risk in all subjects. SV dilatation is a known risk factor for DVT in post-disaster settings. The SV was found to be the most important as the initial site of DVT caused by blood flow stasis [ 17]. The incidence of DVT associated with SV dilatation has also been reported in several studies [12, 18, 19]. Many cases of idiopathic DVT were observed among Japanese people who had a SV diameter $\geq 7 \mathrm{~mm}$. In the disaster affected areas after the Kumamoto Earthquake, DVT was diagnosed more in survivors (staying in disaster shelters) who had an SV diameter $\geq 8 \mathrm{~mm}$ than those with a smaller diameter. In the survivor population of the Great East Japan Earthquake, SV dilatation ( $\geq 9 \mathrm{~mm}$ ) in the DVT-positive group was reported to be significantly higher than that in the DVT-negative group $(\geq 9 \mathrm{~mm})[6,12,18]$. In the patients who underwent arthroplasty, SV dilation ( $\geq 10 \mathrm{~mm}$ ) was reported to be an independent positive predictor of DVT [19]. SV dilatation has not only been reported as a risk factor for DVT, but also as a factor for sustained DVT [20]. SV dilatation contributes to thrombus formation and results in the development of DVT. This may suggest that people with SV dilatation are more likely to suffer from sustained DVT. When physicians treat patients with DVT, they should note that SV dilation is a typical factor in ultrasound imaging that indicates a history of DVT.

In the disaster area, it has been shown that disuse syndrome can diminish calf muscle pump functionality, which causes venous congestion, and people are more likely to develop DVT. In the case of tsunami victims in Ishinomaki City, low levels of physical activity due to their inconvenient lifestyle were observed and this might have contributed to the development of disuse syndrome regardless of living in temporary housing or in their own homes [4]. To avoid having low levels of physical activity, exercise classes were therefore carried out mainly at temporary housing locations. These classes were effective in improving their sense of well-being, but it did not have a substantial impact on the disaster victims [2I]. Our study population might have had exercise habits, but we assume that the reduced levels of physical activity were unable to be prevented. In our walking-hour survey, half of the disaster victims stated that their walking time per day was reduced compared to what it was before the earthquake. SV dilatation caused by reduced levels of physical activity after the disaster may have contributed to the development of DVT.

Our high detection rate of DVT may be due to the fact that this study was conducted in a disaster-affected area and included disaster victims with underlying diseases (hypertension, diabetes mellitus and dyslipidemia). Metabolic syndrome (MetS), the clustering of hypertension, hyperlipidemia, diabetes mellitus, and obesity, is a known factor for increasing the risk of venous thromboembolism (VTE) [22]. In addition, an increased risk of recurrent VTE following the accumulation of MetS components, including hypertension, diabetes mellitus, dyslipidemia and obesity, was reported [23]. The VTE recurrence rate was $14 \%$ in patients with one component, followed by $21 \%$ with two components, $30 \%$ with three components, and $37 \%$ with all four components [23]. The higher the number of MetS components, the higher the risk of VTE.

Disaster-related DVT was not found to disappear even after several years in many people who developed it immediately after the disaster [5, 16]. The reason for this may be because disaster victims with organized thrombi are more likely to suffer from recurrent DVT over a long period of time [6, I6]. SV dilatation has been shown to be a risk factor for DVT in our study, but it was also reported to be a risk factor for sustained DVT. In addition to this, our study has included subjects who developed DVT immediately after the disaster as well as those who had underlying diseases. This may have contributed the high DVT detection rates in our study.

Addressing self-selection and non-respondent biases is necessary in disaster area studies. In this study, there were many subjects who were concerned about disaster-related diseases, which might have introduced self-selection bias, and thus the actual prevalence of DVT might have been lower than that shown in our study. On the other hand, in order to avoid non-respondent bias, it is particularly important to take measures to encourage all survivors to participate in social activities, including screening activities, in the disaster affected areas.

\section{Comparison of background factors between temporary and non-temporary groups}

In the comparison of background factors including those related to living conditions during the disaster reconstruction phase, age, reduction of walking hours and psychological disorders were found to be significantly higher in the temporary housing group than in the non-temporary housing group. In the psychological distress level survey using K6, the prevalence of psychological disorders in both the temporary housing group 
(38.1\%) and non-temporary housing group (22.6\%) were remarkably higher than in the general Japanese population (2.3\%) [24]. A post-disaster survey in Miyagi Prefecture ( 18 years and older) reported that the prevalence of psychological disorders was $8.6 \%$ in disaster public housing and $7.5 \%$ in temporary housing [25]. The prevalence was higher in our study subjects both in temporary and non-temporary housing groups. The rate in another survey $(35.9 \%)$, which was conducted one year after the disaster among residents (40 years and older) in Iwanuma City, Miyagi Prefecture, shows a percentage similar to that of the temporary housing group rate in our study [26]. There may be a gap between the regions within the same prefecture. Because the majority of our subjects belong to the elderly population, the increase in the number of psychological disorders may have been a change specific to the elderly. In the disaster reconstruction phase, stress reactions are likely to cause solitary deaths and social withdrawal among elderly people, and these changes are more common among women [27-29]. However, more than $70 \%$ of the subjects in our study were women in both groups, and this suggests that no difference due to gender may have been observed in the detection rates. Although the risk factors for DVT differ between temporary and non-temporary housing groups, there is no significant difference in the DVT detection rates, which were high in both groups even three years after the disaster. Although there are some differences in terms of support for survivors and their living conditions between the temporary and non-temporary housing groups, it can be assumed that the onset and sustaining of DVT is a health problem related to the lifestyle of individual survivors. In contrast, the risk factors for DVT differ between the temporary and non-temporary groups. Because a previous study reported that risk factors for DVT could change over time after the disaster, the difference in environmental improvement levels might have led to the variation of risk factors $[4,5,16,30]$. The risk factors for DVT in this study differ between the temporary and non-temporary groups depending on the support system and their living conditions, which might have contributed to the high incidence of DVT in both groups.

\section{Risk factors for DVT in the temporary housing group}

In our study, the risk factors for DVT in the temporary housing group were hypertension and the use of sleeping pills. The association between hypertension and DVT was reported in a study conducted six years after the Mid Niigata Prefecture Earthquake [3I]. After a major disaster, the risk of cardiovascular disease, infection and psychological disorders increases among survivors under stressful conditions, and hypertension was the most common incidence [1, 32]. The temporary housing group can be referred to as a high-stress population as they scored $19.1 \pm 9.1$ in the $\mathrm{K} 6$. The use of drugs, such as antipsychotics, and alcohol that depress the central nervous system and cause muscle relaxation of the lower extremity may also contribute to the development of DVT [33, 34]. The longer the evacuation period lasts, the more people complain of mental health problems and insomnia, and they want to take sleeping pills. The use of sleeping pills was reported as one of the risk factors for DVT in a previous study conducted after the Kumamoto Earthquake in 2016 [35]. Because some types of sleeping pills have muscle relaxation effects [36], special attention needs to be paid to prevent the development of DVT. Given these, risk factors for DVT (hypertension and the use of sleeping pills) can be applied to disaster-affected areas in common regardless of the type of disaster. For example, an earthquake directly above its epicenter, tsunami disaster or others.

This study has several limitations. First, this was a cross-sectional study and causality was therefore not determined. Second, a detailed assessment for the stage when our subjects were staying in the disaster shelters was not made. Third, a detailed assessment regarding previously reported common risk factors for DVT was not made. Fourth, because many subjects in the temporary housing group had a history of DVT screening, the awareness of DVT was possibly high in this population. Fifth, it was impossible to target all disaster victims living in temporary housing. Sixth, because medical history and lifestyle were judged using a self-report questionnaire, considerations based on the actual influence were not made. Seventh, those with underlying diseases were also included in our study. Eighth, elderly women tended to participate in community health and social welfare programs more than elderly men. Therefore, less elderly men participated in our study. Future studies that take these eight limitations into consideration are therefore necessary.

\section{Conclusions}

DVT detection rates were similar between the temporary and non-temporary housing groups, and were higher than that in the general Japanese population. The Psychological distress levels of the tsunami victims measured by $\mathrm{K} 6$ were higher in the temporary housing group than in the non-temporary housing group. It is necessary to establish a long-term and awareness-raising disaster victim support system to prevent disaster-related diseases. 


\section{Acknowledgments}

This study was conducted in cooperation with - in no particular order - the medical staff, office workers and students of Yamamoto-cho, Watari-cho, Watarigun Medical Association, Tohoku Fukushi University, the Sendai University Sports Health Science Research and Practice Organization, Fukui Prefectural Hospital, Fukui Kosei Hospital, Fukui-ken Saiseikai Hospital, Takamura Hospital, Fukui Cardiovascular Center, Nakamura Hospital, National Hospital Organization Fukui Hospital, Fukui General Clinic, Tanaka Hospital, Takano Hospital, National Hospital Organization Awara Hospital, National Hospital Organization Tsuruga Medical Center, National Hospital Organization Miyagi National Hospital, Kyoto University Hospital, Harue Hospital, Kaga Medical Center, Anamizu General Hospital, Ishikawa National Hospital, Kanazawa Medical University Hospital, Jouhoku Hospital, Hiroshima City Hiroshima Citizens Hospital, Toyama Rosai Hospital, Toyama City Hospital, Ishinomaki Red Cross Hospital, Morioka Municipal Hospital, Oshu City Mizusawa Hospital, Doijiri Clinic, Fukui University, and the Nippon Dental University. We would like to express our gratitude to them.

\section{Funding details}

This study was funded in 2012 by the Japan Society for the Promotion of Science through the JSPS KAKENHI Grant-in-Aid for Scientific Research Project/Basic Research (C) under Grant [Number 24590685], "Study on the Findings by Lower Limbs Venous Ultrasonography and Hemostatic Test in the Tsunami Disaster Area as a Field."

\section{Conflict of interest}

None.

\section{References:}

I. Kario K. Disaster hypertension - its characteristics, mechanism, and management. Circ J. 2012; 76(3): 553-562, doi: 10.1253/circj.cj-II-1510, indexed in Pubmed: 22327030.

2. Inoue K. Venous thromboembolism in earthquake victims. Disaster Manag Response. 2006; 4(I): 25-27, doi: 10.1016/j. dmr.2005.09.00I, indexed in Pubmed: 16360637.

3. Ueda S, Hanzawa K, Shibata M, et al. High prevalence of deep vein thrombosis in tsunami-flooded shelters established after the great East-Japan earthquake. Tohoku J Exp Med. 2012; 227(3): 199-202, doi: 10.1620/tjem.227.199, indexed in Pubmed: 22728376.

4. Ueda S, Hanzawa K, Shibata M. One-year overview of deep vein thrombosis prevalence in the ishinomaki area since the great East Japan earthquake. Ann Vasc Dis. 2014; 7(4): 365-368, doi: 10.3400/avd.oa. 14-00 106, indexed in Pubmed: 25593620.

5. Shibata M, Chiba H, Sasaki K, et al. The utility of on-site ultrasound screening in population at high risk for deep venous thrombosis in temporary housing after the great East Japan Earthquake. J Clin Ultrasound. 2017; 45(9): 566-574, doi: 10.1002/jcu.22505, indexed in Pubmed: 28556184.

6. Onishi H, Yamamura O, Kosugi I. Comparison of development of deep vein thrombosis detection rates between groups of people in the general shelters and welfare-type shelters in the Kumamoto earthquake area. J Natl Inst Public Heal. 2017; 66: 620-629.

7. Konstantinides SV, Meyer G, Becattini C, et al. ESC Scientific Document Group, The Task Force for the diagnosis and management of acute pulmonary embolism of the European Society of Cardiology (ESC), Authors/Task Force Members, Authors/ /Task Force Members, Task Force for the Diagnosis and Management of Acute Pulmonary Embolism of the European Society of Cardiology (ESC). 2014 ESC guidelines on the diagnosis and management of acute pulmonary embolism. Eur Heart J. 2014; 35(43): 3033-69, 3069a, doi: 10.1093/eurheartj/ehu283, indexed in Pubmed: 25173341.

8. American Institute of Ultrasound in Medicine, American College of Radiology, Society of Radiologists in Ultrasound. Practice guideline for the performance of peripheral venous ultrasound examinations. J Ultrasound Med. 20II; 30(I): 143-150, doi: 10.7863/jum.2011.30.1.143, indexed in Pubmed: 21193718.

9. Murphy TP, Cronan JJ. Evolution of deep venous thrombosis: a prospective evaluation with US. Radiology. 1990; 177(2): 543 -548, doi: 10.1148/radiology.177.2.2217798, indexed in Pubmed: 2217798.

10. Ohgi S, Ito K, Tanaka K, et al. Echogenic Types of Venous Thrombi in the Common Femoral Vein by Ultrasonic B-Mode Imaging. Vascular Surgery. 2016; 25(4): 253-258, doi: 10.1177/I5385744910250040I.

I I. Meissner MH, Moneta G, Burnand K, et al. The hemodynamics and diagnosis of venous disease. J Vasc Surg. 2007; 46 Suppl S: 4S-424S, doi: 10.1016/j.jvs.2007.09.043, indexed in Pubmed: I806856I.

12. Ohgi S, Tachibana M, Ikebuchi M, et al. Pulmonary embolism in patients with isolated soleal vein thrombosis. Angiology. 1998; 49(9): 759-764, doi: 10.1177/000331979804901008, indexed in Pubmed: 9756428.

13. Kessler RC, Andrews G, Colpe LJ, et al. Short screening scales to monitor population prevalences and trends in non-specific psychological distress. Psychol Med. 2002; 32(6): 959-976, doi: 10.1017/s0033291702006074, indexed in Pubmed: |22|4795.

14. Furukawa TA, Kawakami N, Saitoh M, et al. The performance of the Japanese version of the $\mathrm{K} 6$ and $\mathrm{K} 10$ in the World Mental Health Survey Japan. Int J Methods Psychiatr Res. 2008; 17(3): 152-158, doi: 10.1002/mpr.257, indexed in Pubmed: 18763695.

15. Kessler RC, Barker PR, Colpe LJ, et al. Screening for serious mental illness in the general population. Arch Gen Psychiatry. 2003; 60(2): 184-189, doi: 10.1001/archpsyc.60.2.184, indexed in Pubmed: 12578436.

16. Hanzawa K, Ikura M, Nakajima T, et al. Pulmonary embolism or ischemic stroke increase 8-year after mid Niigata Prefecture earthquake 2004 in the residents with asymptomatic belowthe-knee deep vein thrombosis. International Angiology. 2013; 32(Suppl I): 78.

17. Lohr JM, Kerr TM, Lutter KS, et al. Lower extremity calf thrombosis: to treat or not to treat? J Vasc Surg. 1991; 14(5): 618-623, doi: 10.1067/mva.1991.33057, indexed in Pubmed: 1942369. 
18. Maeda F, Yamamura O, Ueda S. Relevant factors for soleal vein dilatation obtained from screening activities in disaster areas. Jap J Med Technol. 2016; 65(I): 25-30.

19. Abe K, Yuda S, Yasui K, et al. Soleal vein dilatation assessed by ultrasonography is an independent predictor for deep vein thrombosis after major orthopedic surgery. J Cardiol. 2017; 69(5): 756-762, doi: 10.1016/j.jjcc.2016.07.004, indexed in Pubmed: 27484506.

20. Shimizu S, Yamamura O, Okamoto H, et al. Study of factors perpetuating deep vein thrombosis in areas affected by the Great East Japan Earthquake. Neurosonology. 2017; 30(2): 43-48, doi: 10.2301/neurosonology.30.43.

2I. Tomata Y, Sato N, Kogure M, et al. [Health effects of interventions to promote physical activity in survivors of the 201। Great East Japan Earthquake. A longitudinal study]. Nihon Koshu Eisei Zasshi. 2015; 62(2): 66-72, doi: I0.1 I 236/jph.62.2_66, indexed in Pubmed: 25865402.

22. Ageno W, Prandoni P, Romualdi E, et al. The metabolic syndrome and the risk of venous thrombosis: a case-control study. J Thromb Haemost. 2006; 4(9): 1914-1918, doi: 10.1 I I //j. 15387836.2006.02 I32.x, indexed in Pubmed: 16848878.

23. Stewart LK, Kline JA. Metabolic syndrome increases risk of venous thromboembolism recurrence after acute deep vein thrombosis. Blood Adv. 2020; 4(I): 127-135, doi: 10.1182/ bloodadvances.201900056I, indexed in Pubmed: 31917844.

24. Sakurai K, Nishi A, Kondo K, et al. Screening performance of $\mathrm{K} 6 / \mathrm{KIO}$ and other screening instruments for mood and anxiety disorders in Japan. Psychiatry Clin Neurosci. 201 I; 65(5): 434-44I, doi: 10.1 III/j.1440-1819.2011.02236.x, indexed in Pubmed: 21851452.

25. Miyagi Prefectural Office, Health Promotion. Section: disaster public housing resident health findings; 2015. (in Japanese). https:// www.pref.miyagi.jp/uploaded/attachment/36632I.pdf (I.04.2019).

26. Koyama S, Aida J, Kawachi I, et al. Social support improves mental health among the victims relocated to temporary housing following the Great East Japan Earthquake and Tsunami. Tohoku J Exp Med. 2014; 234(3): 24I-247, doi: 10.1620/tjem.234.24I, indexed in Pubmed: 2538228।.

27. Jia Z, Tian W, Liu W, et al. Are the elderly more vulnerable to psychological impact of natural disaster? A population-based survey of adult survivors of the 2008 Sichuan earthquake. BMC Public Health. 2010; 10: 172, doi: 10.1 186/1471-2458-10-172, indexed in Pubmed: 20353554.

28. Zhang Y, Ho SMY. Risk factors of posttraumatic stress disorder among survivors after the 512 Wenchuan earthquake in China. PLoS One. 201I; 6(7): e2237I, doi: 10.1371/journal. pone.002237I, indexed in Pubmed: 21799838.

29. Farooqui M, Quadri SA, Suriya SS, et al. Posttraumatic stress disorder: a serious post-earthquake complication. Trends Psychiatry Psychother. 2017; 39(2): 135-143, doi: 10.1590/22376089-2016-0029, indexed in Pubmed: 28700042.

30. Hasegawa K, Yoshino H, Yanagi $U$, et al. Indoor environmental problems and health status in water-damaged homes due to tsunami disaster in Japan. Building and Environment. 2015; 93: 24-34, doi: 10.1016/j.buildenv.2015.02.040.

31. Hanzawa K, Okamoto T, Sato K, et al. DVT in residents in mid Niigata Prefecture earthquake after 6 years: relationship between DVT and hypertention. The Japanese Journal of Phlebology. 20I2; 23(4): 3I5-320, doi: 10.7I34/phlebol.23.3I5.

32. Kario K, Matsuo T, Kobayashi H, et al. Earthquake-induced potentiation of acute risk factors in hypertensive elderly patients: possible triggering of cardiovascular events after a major earthquake. J Am Coll Cardiol. 1997; 29(5): 926-933, doi: 10.1016/ s0735-1097(97)00002-8.

33. Jönsson AK, Schill J, Olsson H, et al. Venous Thromboembolism During Treatment with Antipsychotics: A Review of Current Evidence. CNS Drugs. 2018; 32(I): 47-64, doi: 10.1007/s40263018-0495-7, indexed in Pubmed: 29423659.

34. Karino T, Motomiya M. Flow through a venous valve and its implication for thrombus formation. Thromb Res. 1984; 36(3): 245-257, doi: 10.1016/0049-3848(84)90224-x, indexed in Pubmed: 6515603 .

35. Sato K, Sakamoto K, Hashimoto Y, et al. KEEP Project. Risk Factors and Prevalence of Deep Vein Thrombosis After the 2016 Kumamoto Earthquakes. Circ J. 2019; 83(6): 13421348, doi: 10.1253/circj.CJ-18-1369, indexed in Pubmed: 30956268.

36. Gaillard. JM. Comparison of two muscle relaxant drugs on human sleep: diazepam and parachlorophenylgaba. Acta Psychiatr Belg. 1977; 77(3): 410-425. 